Assurances et gestion des risques

Insurance and Risk Management

\title{
L'assurance sans assureur ou le P2P
}

\section{Michel Turcotte}

Volume 84, numéro 1-2, 2017

URI : https://id.erudit.org/iderudit/1041821ar

DOI : https://doi.org/10.7202/1041821ar

Aller au sommaire du numéro

\section{Éditeur(s)}

Faculté des sciences de l'administration, Université Laval

\section{ISSN}

1705-7299 (imprimé)

2371-4913 (numérique)

Découvrir la revue

Citer ce document

Turcotte, M. (2017). L'assurance sans assureur ou le P2P. Assurances et gestion des risques / Insurance and Risk Management, 84(1-2), 77-87.

https://doi.org/10.7202/1041821ar

Tous droits réservés $\odot$ Faculté des sciences de l'administration, Université Laval, 2017
Ce document est protégé par la loi sur le droit d'auteur. L'utilisation des services d'Érudit (y compris la reproduction) est assujettie à sa politique d'utilisation que vous pouvez consulter en ligne.

https://apropos.erudit.org/fr/usagers/politique-dutilisation/ 


\section{L'ASSURANCE SANS ASSUREUR OU LE P2P}

Par : Michel Turcotte 1

On entend beaucoup parler ces derniers temps d'assurance sans intermédiaire, une forme d'autofinancement impliquant uniquement des assurés, pas d'assureur. C'est ce que les anglophones appellent le «Peer to Peer» ou P2P. Sommes-nous à l'aube d'une véritable révolution du modèle d'assurance?

\section{Le P2P en Assurance}

Un modèle de P2P pur dans le monde de l'assurance implique des individus qui décident de s'assurer entre eux sans l'intermédiaire d'un assureur ou d'une autre forme de tiers centralisateur.

Par exemple, un groupe de dix cyclistes qui décident d'assurer le vol de leur vélo par les autres membres du groupe. D'un commun accord, chacun s'engage à indemniser celui qui sera victime d'un vol. Pas besoin de verser une prime initiale, l'indemnisation se ferait au moment du vol et au prorata de la valeur des vélos. Si tous les vélos valent $1000 \$$, alors chacun des membres y compris la victime versera $10 \%$ ou $100 \$$. Si un vélo vaut $500 \$$ alors que les autres valent $1000 \$$ alors un individu payera $5,3 \%$ de la perte $(500 / 9500)$ et les autres payeront $10,5 \%(1000 / 9500)$.

Les années sans vol, aucun membre du groupe n'est tenu de payer quoi que ce soit, ce qui rend, en moyenne sur une longue période, le coût par individu assez faible. Il n'y a pas de frais d'expert en sinistre pour évaluer la perte, pas de frais d'administration ou de profit d'un assureur. Il n'y a tout simplement pas d'intermédiaire. 
Dans l'exemple précédent, puisque le mécanisme est assez simple et que les dix membres du groupe peuvent se connaitre et se faire confiance, cela peut fonctionner. Toutefois, si ces mêmes individus décidaient d'assurer leur habitation, la garantie se complexifie. En effet, avec une valeur moyenne des maisons à Montréal de $336050 \$ 2$, un incendie qui détruirait totalement une d'entre d'elles couterait 33605 \$ à chacun des propriétaires de la mutualité. Peut-on être confiant que chacun sera en mesure de payer?

\section{LE MODÈLE P2P PUR EST-IL POSSIBLE?}

L'exemple de l'assurance habitation, illustre bien que, pour certains risques, il faudra inclure un plus grand nombre de membres dans le groupe pour amortir l'impact financier d'une perte. Se pose alors une difficulté majeure liée à un manque de confiance envers un nombre important de souscripteurs qui ne se connaissent pas.

Un des rôles de l'assureur est justement de mitiger ce risque, de réduire l'incertitude au regard de l'économie du contrat. L'assureur perçoit des primes et accumule des surplus pour améliorer sa situation financière afin que les assurés aient confiance en sa capacité de payer les pertes.

Le gestionnaire de risques pourrait-il avoir le même niveau de confort ou de confiance envers un groupe d'inconnus qui s'uniraient afin d'offrir une garantie d'assurance?

Nous assistons, de nos jours, à un développement technologique qui pourrait avoir trouvé une réponse à cette question, une solution pour contrer cette incertitude et mitiger ce risque.

En effet, ce besoin de réduire l'incertitude s'applique également en ce qui concerne la monnaie. Nous avons confiance au dollar canadien parce que nous avons confiance aux institutions qui en garantissent la valeur. De nos jours, il existe une monnaie sans garantie d'aucune institution et dont la valeur surpasse celle de l'once d'or: le Bitcoin.

La technologie derrière la conception de cette monnaie électronique pourrait être utilisée pour toute sorte de fins, incluant l'assurance.

Donc avant de conclure que l'assurance "peer-to-peer" est une utopie, nous devons faire une petite incursion dans le monde des technologies pour aborder le sujet des Blockchains. 


\section{BLOCKCHAIN}

Un mot inconnu pour certains et obscur pour d'autres mais certainement le plus grand «buzz» dans le monde des technologies. Le terme blockchain a été utilisé pour la première fois dans un livre blanc portant sur le sujet du Bitcoin et écrit par Satoshi Nakamoto, qui est en fait le pseudonyme d'une personne ou d'un groupe de personnes. Depuis, des spécialistes s'entendent pour dire que c'est la plus grande révolution technologique depuis internet.

Sans avoir l'intention de présenter une analyse exhaustive de ce concept, quelques mots suffiront pour une brève description.

Imaginez un système intégré ou tous les ordinateurs sont reliés entre eux. Chaque fois qu'une transaction est effectuée, tous les détails sont enregistrés dans un block qui est ensuite encrypté. Tous les blocks sont reliés les uns aux autres par une chaine. Ainsi, l'entièreté du détail de toutes les transactions devient disponible à tous les utilisateurs. Ceux-ci peuvent en lire le contenu mais celui-ci ne peut ni être détruit ni être modifié.

La technologie blockchain existe depuis 2008. Elle constitue l'architecture sous-jacente de la monnaie numérique bitcoin. Cependant, depuis quelques années, plusieurs individus et organisations participent au développement de son utilisation dans divers domaines.

La blockchain se veut donc une forme de base de données qui permet le stockage et la transmission d'information de façon transparente mais surtout de façon sécuritaire. Le plus important, c'est que le tout se fait sans aucune intervention d'une forme de contrôle centralisée. La base de données contient ainsi tout l'historique des échanges ou transactions et ses données sont partagées par l'ensemble des utilisateurs qui peuvent ainsi vérifier la validité de la chaine.

Un des compétiteurs du bitcoin est l'Ether. Sa plateforme s'appelle l'Ethereum. C'est sur cette plateforme que plusieurs développeurs s'acharnent pour révolutionner le monde de demain. ${ }^{3}$

Un des développements prometteurs est l'utilisation de la blockchain avec des smart contracts; des programmes autonomes capables d'exécuter les conditions du contrat, rendant tout intermédiaire caduque. 
La prochaine version d'Ethereum, intitulée "Metropolis " ${ }^{4}$, consistera en un lancement grand public permettant aux utilisateurs non-avertis de bénéficier d'une interface utilisateur quasi complète. Ce sera la première version offerte au grand public, les versions actuelles d'Ethereum étant réservées aux initiés. La date de sortie est toutefois inconnu au moment d'écrire ces lignes.

\section{LA BLOCKCHAIN ET L'ASSURANCE}

La combinaison de la blockchain avec les smart contracts permettent la mise en place d'organisations décentralisées autonomes (DAO). Ce sont des entités autonomes dans la blockchain, sans statut juridique formel. Leurs règles de fonctionnement sont inscrites dans du code informatique. Dans le cas de l'assurance, ces DAO peuvent être utilisées pour créer des groupements d'assurés, sur le format P2P, sans organisation centrale de contrôle comme un assureur, chaque groupe étant gouverné par les assurés eux-mêmes.

Ce modèle collaboratif permet un déplacement du pouvoir de décision d'un assureur vers les assurés qui, par un système de vote, peuvent décider collectivement de la validité d'une réclamation et du mode de redistribution des surplus.

\section{IMPACT SUR LE MARCHÉ DE L'ASSURANCE}

Nous observons l'émergence d'une multitude de sociétés startups qui prétendent constituer une véritable révolution dans le monde de l'assurance grâce à ce concept de P2P.

Dans les faits, la plupart d'entre elles propose un mode de fonctionnement qui se résume ainsi: les assurés versent une prime à une entité centrale, l'unité centrale prend un pourcentage de la prime pour ses frais, verse une part de la prime à un réassureur, utilisent l'excédent pour payer les pertes courantes et retourner les surplus aux assurés.

Cette description, loin d'être révolutionnaire, innovante ou nouvelle, s'apparente grandement à un vieux modèle: la mutualisation.

Les mutuelles existent depuis des lunes. Il suffit de penser à la Hand in Hand Fire \& Life Insurance Society qui fût fondé en 1696 à Londres. 
Dans les faits, plusieurs de ces nouvelles sociétés startups, qui cherchent une place dans le marché des assurances, ne présentent pas un modèle de P2P pur, ni même un modèle nouveau. Par contre, elles intègrent un modèle existant sur une nouvelle architecture à la fine pointe de la technologie qui pourrait permettre aux assureurs d'accélérer le processus de souscription et de règlement des sinistres, d'améliorer la transparence et de réduire les coûts de gestion.

Évidemment, toutes ces startups ne proposent pas le même modèle.

\section{VÉRITABLE ASSURANCE P2P}

Parmi les rares véritables modèles $\mathrm{P} 2 \mathrm{P}$ on retrouve la startup Russe Teambrella ${ }^{5}$ qui fait sa publicité en se vantant de ne pas être de l'assurance, mais bien plus! Elle veut offrir une assurance juste, transparente et abordable. Il n'y a pas de mise en commun comme chez les autres assurances, c'est du pur P2P. Le site présente un excellent livre blanc ${ }^{6}$ qui explique le fonctionnement de cette assurance. L'inconvénient de ce modèle où les membres votent pour décider si la perte est indemnisable, en tout, en partie ou pas du tout, est qu'il est fort possible de ne pas être entièrement indemnisé.

En début de texte, il était question d'un exemple avec des cyclistes qui s'unissent pour s'auto-assurer. C'est un peu ce qu'offre la société Cycle Syndicate ${ }^{7}$. Avec cette société basée à Londres, il faut payer une prime au départ et la société retient $15 \%$ de frais d'administration.

\section{LES P2P MUTUELLES}

La société Française Inspeer ${ }^{8}$ a lancé un produit qui se définit comme une assurance collaborative pour l'assurance automobile des véhicules électriques en France.

Fondée en Février 2014, Inspeer se définit comme la première solution d'assurance collaborative proposant aux particuliers d'optimiser leurs contrats en s'appuyant sur une communauté solidaire et responsable. Le produit d'assurance couvre le dommage, la responsabilité, la location de batterie (particularité en France) et le remorquage suite à un accident. Une partie des cotisations (primes) va à l'assureur pour les gros sinistres tandis qu'une autre partie va dans un fonds collaboratif dédié aux assurés. À la fin de l'année, s'il reste de l'argent dans le fonds, il est restitué à chacun des assurés sous forme de ristourne. 
La société allemande Friendsurance9 fondée en 2010 reproche aux assureurs le coût élevé et le manque de transparence. C'est pourquoi elle propose un concept de "peer-to-peer" qui se veut révolutionnaire parce qu'il récompense le membre d'un petit groupe d'utilisateurs s'ils n’ont pas de perte dans l'année.

Ce modèle propose le versement de $60 \%$ de la prime à l'assureur (ou une forme de réassurance) et $40 \%$ dans un fonds qui sert à verser une ristourne aux assurés si le petit groupe n'a pas eu de réclamation.

Au Royaume-Uni, la société Heyguevara ${ }^{10}$ offre une assurance automobile. Son introduction dit que les vieilles assurances sont des ordures (Rubbish) alors que Guevara est $100 \%$ nouveau et non-ordurier (traduction libre).

Le fonctionnement consiste en un assuré joignant un groupe, il paie une prime dont une partie sert à l'assurance et l'autre va dans un fonds. Les pertes importantes sont payées par l'assurance, les plus petites par le fonds. À la fin de l'année, si le niveau de perte est bas, les membres du groupe se partagent le contenu du fonds.

La société d'assurance P2P Dynamis ${ }^{11}$, quant à elle, cherche à proposer un produit d'assurance chômage. Elle promet une meilleure transparence grâce aux smart contracts et sa plateforme Ethereum. On retrouve sur leur site un intéressant livre blanc ${ }^{12}$ qui explique en détail ce projet.

La société française WeKeep ${ }^{13}$ se présente en disant: «Et si vous n'aviez plus besoin d'assureurs pour les assurances non obligatoires " et se définite comme une mutuelle décentralisée. Elle proposera un modèle où les transactions seront enregistrées dans une blockchain et ce sont les assurées qui décideront si une réclamation est payable ou pas. Le projet est actuellement en développement.

La société Pontu ${ }^{14}$ se présente comme la première société d'assurance voyage P2P au monde. Fondée en 2016, elle promet une assurance simple et non dispendieuse. Les voyageurs payent une prime au départ. Pontu crée des groupes avec des voyageurs aux caractéristiques similaires. De la même façon que les précédentes, si les sommes accumulées dans le fonds ne sont pas entièrement utilisées pour les diverses indemnisations, elles sont redistribuées entre les membres contributeurs. 


\section{LES P2P PLUS IMPLANTÉES}

La société chinoise TongJuBao ${ }^{15}$, qui signifie se protéger ensemble, est en opération depuis plus d'un an et revendique plus de 10000 membres.

Elle propose une l'assurance pour des risques sociaux ou familiaux telle qu'une assurance contre le divorce. Un assuré paye 400 yuans de droit d'entrée minimum et peut recevoir 1200 yuans par mois pendant un an en cas de divorce. Elle offre aussi une assurance kidnapping d'enfant. Pour 288 yuans de mise de départ, l'assuré bénéficie d'une prestation de 100000 yuans pour inciter des enquêteurs à procéder plus rapidement à la recherche d'indices.

La plus connu de ces nouvelles sociétés est certes Lemonade ${ }^{16}$. Son CEO et co-fondateur, Daniel Schreiber participe à de multiples conférences pour vanter les mérites de son modèle.

Lemonade se définit comme une compagnie d'assurance biens et responsabilité qui transforme complètement le modèle d'assurance traditionnel en mettant le focus sur la technologie et la transparence. Puisque Lemonade est rémunérée par des revenus fixes, elle n'a aucun intérêt à rejeter les réclamations ou en retarder le paiement.

Son slogan est tout simplement: "Oubliez tout ce que vous savez sur l'assurance" et son modèle inclut une forme de réassurance pour $20 \%$ de la prime reçue. Selon son site web, les réassureurs incluent plusieurs syndicats des Lloyds, XL Catlin et autres.

En ce qui concerne la notation financière de cet assureur, il prétend être noté A- Exceptionnel par Demotech Inc $^{17}$.

Lemonade offre une solution d'assurance pour les biens d'un locataire ou d'un propriétaire. Ses promesses sont un délai de 90 secondes pour être assuré et de trois minutes pour être indemnisé. En fait, on prétend inverser le modèle d'assurance traditionnel en traitant les primes payées comme l'argent de l'assuré et non de l'assureur. Lemonade charge $20 \%$ de la prime pour la gestion du système et, en échange, on promet de payer les réclamations rapidement et de verser les surplus à une ouvre caritative choisie par l'assuré. 


\section{RÉACTION DES ASSUREURS}

Les assureurs suivent ces avancées technologiques de près. Certaines sociétés indépendantes travaillent au développement de solutions pour les assureurs.

C'est le cas de la société Guardtime ${ }^{18}$ qui offre déjà une solution basée sur sa propre technologie KSI qui aide les assureurs à mieux opérer. Les services offerts incluent le support dans l'émission des polices, la gestion des réclamations, la subrogation et la conformité réglementaire.

La société américaine IBM, dans son laboratoire de Zurich, en Suisse, travaille sur le projet Hyperledger ${ }^{19}$ avec plus d'une centaine de sociétés afin de développer une blockchain pour entreprise qui simplifiera les transactions, réduira les coûts d'intermédiaire et assurera l'intégrité des transactions.

Certains assureurs investissent dans des sociétés de technologies comme AXA Strategic Ventures qui a investi 55M\$dans la société Blockstream, un leader dans le secteur des blockchains.

Blockstream est une jeune startup fondée en 2014 et installée à Montréal. Son domaine d'expertise, la blockchain. Blockstream est à la pointe de cette technologie, et a développé Sidechains, un protocole blockchain capable de mettre en place des réseaux publics et privés pouvant interagir les uns avec les autres, et ainsi d'assurer des transactions agiles et sécurisées. Blockstream pourrait fournir aux sociétés la solution blockchain la plus mature et sécurisée du marché, réduisant le coût de la confiance ainsi que la fragmentation du réseau tout en renforçant les standards de sécurité.

D'autres assureurs sont activement impliqués dans cette quête technologique. C'est le cas du réassureur Swiss $\operatorname{Re}^{20}$ et quatre autres assureurs qui ont lancé, en octobre 2016, le projet B3i qui vise à explorer comment la technologie blockchain peut être utilisée pour mieux servir leurs clients. Le succès d'un tel projet pourrait permettre de réduire les échanges papiers, de simplifier les contrats de réassurance et accélérer le flux d'information et d'argent.

En février 2017, une dizaine d'autres assureurs et réassureurs ont joint B3i pour lui conférer un statut international avec des membres de l'Asie, de l'Europe et de l'Amérique. Parmi les membres ont retrouvent: Achmea, Aegon, Ageas, Allianz, Generali, Hannover Re, Liberty Mutual, MunichRe, RGA, SCOR, Sompo Japan Nipponkoa Insurance, SwissRe, Tokio Marine Holdings, XL Catlin and Zurich Insurance Group. 


\section{LES DÉFIS}

Avant de voir toutes ces sociétés startups venir perturber le marché de l'assurance, il y a plusieurs défis à surmonter.

Le premier défi concerne la règlementation. Plusieurs types d'assurance sont strictement règlementés par les autorités. Prenons l'exemple de l'assurance automobile au Canada ou l'assurance Worker Compensation aux États-Unis. Ces assurances sont obligatoires et doivent répondre à des critères bien précis. Les autorités accepteront-elles des modèles d'assurance collaborative?

La même question se pose lorsque l'assurance est exigée par un prêteur. L'entente de prêt liste les assurances requises et leurs conditions. Les prêteurs accepteront-il ces nouvelles formes d'assurance?

D'un point de vu d'un gestionnaire de risques, l'absence de "confiance" envers les inconnus que la blockchain vise à corriger n'est pas la seule préoccupation.

Les termes et conditions de la police d'assurance, en d'autres mots les garanties conférées, sont souvent d'une forme manuscrite adaptée aux besoins particulier de cet assuré. Les modèles qui semblent émerger pour l'instant visent des garanties bien standardisées.

Plusieurs gestionnaires de risques ont des politiques corporatives au sujet des assurances qu'ils doivent respecter. Parmi celles-ci on retrouve une notation au sujet de la stabilité financière émise par des sociétés réputées tels qu'A.M. Best, Fitch ou Standard \& Poor. Les assurances de type collaboratif, sans intermédiaire, pourront-elles se conformer à cette exigence?

Dans bien des cas, les gestionnaires de risques doivent assurer des activités ou des actifs répartis dans plusieurs pays. Comment ces nouveaux modèles d'assurance répondront-ils à ce besoin?

Enfin, le but premier de l'achat d'une assurance par un gestionnaire de risques est de transférer un risque à un assureur afin d'être certain d'obtenir une indemnisation en cas de sinistre. Les modèles collaboratifs proposent souvent que les membres décident entre eux si une perte est payable en tout, en partie ou pas du tout. On transfère donc la décision d'indemniser ou pas entre les mains des membres qui ne sont pas nécessairement aptes à en juger. Pour deux pertes similaires, il est possible d'avoir deux formes d'indemnisation bien différentes selon l'opinion de membres non-formés pour prendre une telle décision. 
Ceci dit, même la blockchain, qui doit introduire un haut niveau de confiance, peut subir des contrecoups. Ce fut le cas avec le projet TheDAO qui a été victime de hackers.

The DAO était un projet d'autofinancement ou les investisseurs mettaient leurs fonds en commun et ensuite votaient sur les projets dans lesquels The $\mathrm{DAO}^{21}$ souhaitaits investir. Plus de 11000 membres ont investi en Ether l'équivalent de $150 \mathrm{M} \$$ et devaient voter sur 50 projets qui avaient été soumis. Mais le 18 juin 2016, quelques semaines après son lancement, des pirates sont parvenus à voler 3,6M d'Ether, ce qui a provoqué l'effondrement du projet et a soulevé de sérieuses questions sur l'efficacité de ce type de blockchain.

\section{CONCLUSION}

Il est fort probable que dans un avenir rapproché nous assistions à un changement opérationnel chez les assureurs traditionnels. L'intégration des technologies blockchain pourrait réduire les coûts d'opération, accélérer le processus de souscription et possiblement améliorer le processus de gestion des réclamations.

Par contre, il serait fort étonnant d'assister à l'émergence de modèles alternatifs qui viennent révolutionner ou perturber le marché de l'assurance actuel. 


\section{NOTES}

1. Michel Turcotte détient un MBA de l'université HEC Montréal. II est directeur principal risques et assurances chez Ivanhoé Cambridge, la filiale immobilière de la Caisse de dépôt et de placement du Québec et il enseigne la gestion de risques à l'université McGill.

2. Aperçu du marché de l'immobilier résidentiel 2016 par Remax

3. Voir la DevCon2, grand rassemblement annuel de la communauté de la blockchain Ethereum qui s'est déroulé à Shanghaï du 19 au 21 septembre 2016.

4. http://www.coindesk.com/vitalik-buterin-issues-update-ethereums-metropolis-upgrade/

5. https://teambrella.com/

6. https://teambrella.com/WhitePaper.pdf

7. http://cyclesyndicate.co.uk

8. http://inspeer.me/

9. http://www.friendsurance.com/

10. https://heyguevara.com/

11. http://dynamisapp.com/

12. http://www.dynamisapp.com/whitepaper.pdf

13. http://wekeep.io/

14. http://pontu-insurance.wixsite.com/travel

15. http://www.tongjubao.com/en

16. https ://www.lemonade.com/

17. http://www.demotech.com/

18. https://guardtime.com/

19. https://www.hyperledger.org/?cm_mc_uid=58933499492314921776287\&cm_mc_ sid_50200000 $=1492451968$

20. http://www.swissre.com/reinsurance/insurers_and_reinsurers_launch_blockchain_initiative.html 